\title{
Hierarchical Optical Memory System Using Near- and Far-field Accesses
}

\author{
Makoto Naruse \\ National Institute of Information and Communications Technology, 4-2-1 Nukui-kita, Koganei, Tokyo 184-8795, Japan \\ Takashi Yatsui \\ Japan Science and Technology Agency, 687-1 Tsuruma, Machida, Tokyo 194-0004, Japan \\ Wataru Nomura and Motoichi Ohtsu \\ University of Tokyo, 7-3-1 Hongo, Bunkyo-ku, Tokyo 184-8795, Japan
}

\begin{abstract}
We propose a hierarchical optical memory system in which near-fields and far-fields read detailed dipole distributions and features within a region-of-interest, respectively. With hierarchical coding, near- and far-field accesses are associated with different hierarchical information.

(C)2005 Optical Society of America

OCIS codes: (210.0210) Optical data storage; (210.4680) Optical memories; (070.6020) Signal processing
\end{abstract}

\section{Introduction}

Ultrahigh-capacity optical data storage is an important technology. Various methods to increase the storage density have been pursued, such as shortening the operating wavelength [1]. With such methods, the storage density is still bound by the diffraction limit of light. One technique to overcome this limitation, which we make use of in our proposed system, is optical near-fields [2]. These high-density optical memories, however, need certain seeking or scanning mechanisms, which might be a problem, for instance, when searching terabyte- or petabyte-scale memories.

In dealing with this problem, we first note that information has hierarchy in terms of its meaning or quality, such as "abstract" and "detailed" information, "low" and "high" resolution information, and so forth. Similarly, as discussed below, we can find physical hierarchy in the different modes of light propagation. For example, in a nearfield, a spatial distribution of the dipole moments is obtained, whereas in a far-field, the macroscopic features of the dipole moments are obtained. We associate these hierarchies in the system demonstrated in this paper, that is, a hierarchical optical memory system having both near- and far-field readout functions with a simple digital coding scheme. As schematically shown in Fig. 1, in the far-field mode, low-density, rough information is read-out, whereas in the near-field mode, high-density, detailed information is read-out.

\section{Logical model of hierarchical coding}

The two-layer hierarchical memory in this paper is explained using the notations far-code and near-code. The farcode depends on the array of bits distributed within a certain area and is determined logically to be either ZERO or ONE. Each far-code is comprised of multiple smaller-scale elements, whose existence is determined by the nearcode. To obtain such information hierarchically, we introduce the following simple logical model.

Consider an $(N+1)$-bit digital code, where $N$ is an even number. Now, let the far-code be defined depending on the number of ONEs (or ZEROs) contained in the $(N+1)$-bit digital code:

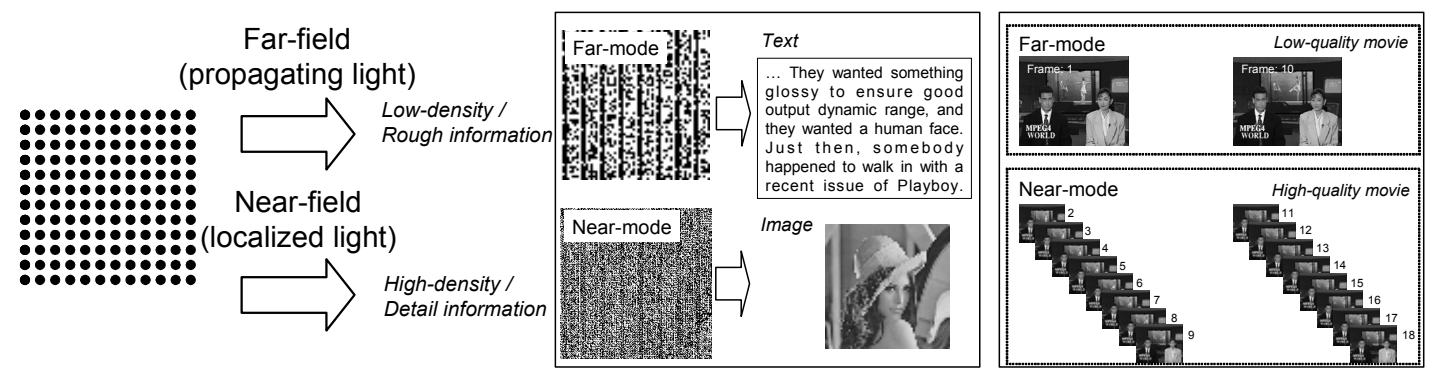

Fig. 1 Hierarchical optical memory using near- and far-field accesses. 


$$
\text { far }- \text { code }=\left\{\begin{array}{cc}
1 & \text { If the number of ONEs } \geq N / 2 \\
0 & \text { otherwise }
\end{array} .\right.
$$

The $(N+1)$ digits provide a total of $2^{N+1}$ possible different permutations, or codes. Here, we note that half of them, namely $2^{N}$ permutations, have less than $N / 2+1$ ONEs among the $(N+1)$ digits (i.e., far-code $\left.=1\right)$, and the other half, also $2^{N}$ permutations, have more than $N / 2+1$ ZEROs (i.e., far-code $=0$ ). In other words, $2^{N}$ different codes could be assigned to two $(N+1)$-bit digital sequences so that their corresponding far-codes are ZERO and ONE, respectively. We call this $(N+1)$-bit code a near-code.

In Fig. 2, example near-codes are listed when $N=8$. The correspondence between $2^{N}$ original codes and the $(N+1)$-bit near-codes is arbitrary. Therefore, we need a table-lookup when decoding an $(N+1)$-bit near-code to the original code. The example near-codes shown in Fig. 2(a) are listed in ascending order, but other lookup-tables or mappings are also possible.

Fig. 2(b) schematically demonstrates example codes in which a 9-bit near-code is represented in a $3 \times 3$ array of circles, where black and white mean ONE and ZERO, respectively. Here, (1) if the number of ONEs in the nearcode is larger than five, then the far-code is ONE; and (2) if the number of ONEs in the near-code is four or less, then the far-code is ZERO.

Suppose, for example, that the far-code stores text data and the near-code stores 256-level (8-bit) image data. Consider a situation where the far-code should represent an ASCII code for "A", whose binary sequence is "0100001". Here, we assume that the gray levels of the first two pixels, which will be coded in the near-code, are the same value. (Here, they are at a level of "92".) However, the first two far-codes are different (ZERO followed by ONE). Referring to the rule shown in Fig. 2(a), and noticing that the first far-code is ZERO and the near-code should represent "92", the first near-code should be "001101010". In the same way, the second near-code is "110001011", so that it represents the level "92", while its corresponding far-code is ONE.

\section{Physical model of the near- and far-codes}

The far-code is determined based on the rule given by eq. (1), which depends on the number of ONEs coded in the near-code. Here, we employ a simple physical model where the near-code is represented by an array of dipole moments. As schematically shown in Fig. 3(a), dipole moments are distributed in an $x y$ plane, where an $(N+1)$-bit code is assigned in an equally spaced grid. The electrical field at position $\boldsymbol{r}$ in Fig. 3(a) is given by

$$
E(\boldsymbol{r})=\sum_{i, j} E_{i, j} e^{-i \omega t+i k\left|\boldsymbol{r}-\boldsymbol{s}_{i, j}\right|} \frac{1}{\left|\boldsymbol{r}-\boldsymbol{s}_{i, j}\right|}
$$

where $\omega$ is the operating frequency, $k$ is the wave number, and $s_{i, j}$ represents the position of a dipole specified by indexes $i$ and $j$ [3]. The existence of the dipole at the position $s_{i, j}$ is given by the near-code as

$$
E_{i, j}=\left\{\begin{array}{cc}
0 & \text { nearcode }(i, j)=0 \\
E_{0} & \text { nearcode }(i, j)=1
\end{array}\right.
$$

Suppose that the pitch of adjacent dipoles is given by $b$. Here, if we assume that $b<<1<<r$, then eq. (2) is simplified

(a)

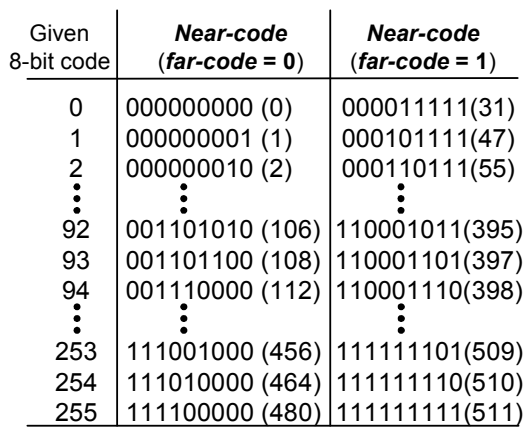

(b)
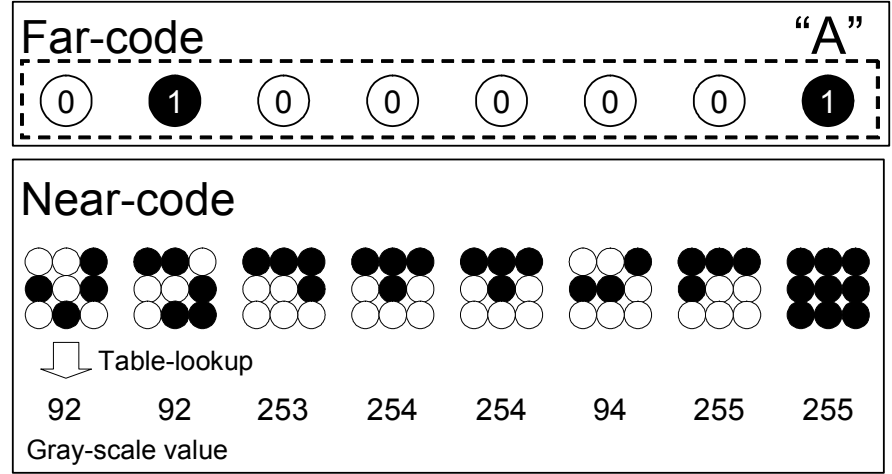

Fig. 2 Example of logical model for the near-code and far-code. Here, the original 8-bit information is coded differently in near-code depending on its corresponding far-code which is either ZERO or ONE. 
to

$$
E(\mathbf{r})=E_{0} \frac{e^{-i \omega t+i k r}}{r} \sum_{i, j} \text { nearcode }(i, j)
$$

which means that the electrical field intensity at position $\boldsymbol{r}$ is proportional to the number of ONEs given by the nearcode in that area.

\section{Experiment and Simulation}

For the experiment, an array of particles was made, in which 100-nm-diameter Au particles were distributed over a $\mathrm{SiO}_{2}$ substrate. Each group of $3 \times 3 \mathrm{Au}$ particles with $300-\mathrm{nm}$ pitch represented a near-code, and adjacent nearcodes were located with 2- $\mu \mathrm{m}$ spacing. An SEM picture is shown in Fig. 3(b-1). These particles were fabricated by using electron-beam (EB) lithography using a $\mathrm{Cr}$ buffer layer (a liftoff technique), which allowed Au formation on the $\mathrm{SiO}_{2}$ substrate with features having a diameter down to $35 \mathrm{~nm}$, as shown in Fig. 3(b-2). Although final experimental results are still pending, basic simulations were performed assuming ideal isotropic metal particles to see how the scattering light varies depending on the number of particles for the far-code using a Finite Difference Time Domain simulator (Fujitsu Inc. Poynting). Fig. 3(c) shows calculated scattering cross sections as a function of the number of particles. The assignment of particle(s) in the grid is also shown. A linear correspondence to the number of particles was observed.

\section{Summary}

In summary, we propose a hierarchical optical memory system in which near-fields are used to read detailed dipole distributions, whereas far-fields are used to detect features within a region-of-interest. An experimental device and simulations were also shown. With hierarchical coding, near- and far-field accesses are associated with different hierarchical information, which should help overcome problems involved in searching huge memory spaces. General design of the logical model and applications will also be pursued as well as physical implementations.

\section{References}

[1] For example, http://www.blu-ray.com/

[2] M. Ohtsu, K. Kobayashi, T. Kawazoe, S. Sangu, and T. Yatsui, "Nanophotonics: design, fabrication, and operation of nanometric devices using optical near fields,” IEEE J. Select. Topics Quantum Electron. 8, 839-862 (2002).

[3] M. Ohtsu and K. Kobayashi, Optical Near Fields (Springer, 2004), Chap. 4.

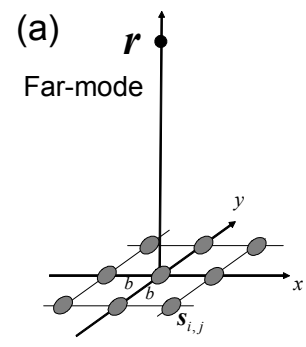

Near-mode

$(\mathrm{b}-1)$

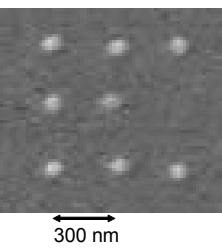

$(b-2)$

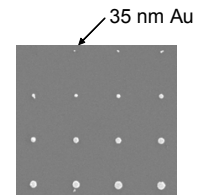

(c)

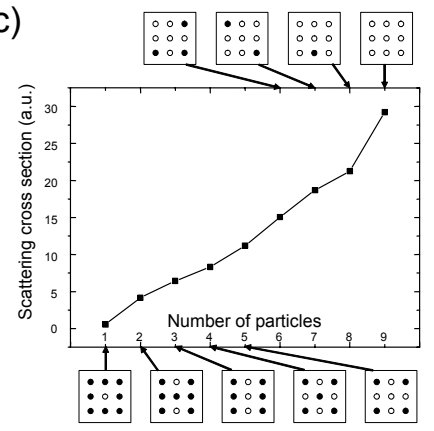

Fig. 3 (a) Physical model. (b) Au particle arrays for the experiment. (c) Simulation of the far-code. 